\title{
234 Casos de Embarazos Múltiples
}

\author{
Dr. Luis A. Sánchez R.
}

Del Instituto Materno-Infantil de Bogotá

El presente trabajo tiene por objeto revisar la estadística de los casos de embarazo múltiple que se han presentado en los dos últimos años en el Instituto Materno Infantil "Concepción Villaveces de Acosta" y comparar estas cifras con las presentadas en trabajos de otros países. Esta revisión la consideramos de valor pues es la segunda que se hace al respecto entre nosotros, siendo la primera la del Dr. Leopoldo Zerda Jiménez, como tesis de grado en 1948. (13)

\section{EMBARAZOS MULTIPLES}

Revisión de 234 casos

En el período de 2 años comprendido entre el $1^{\text {o }}$ de Marzo de 1958 y el 29 de Febrero de 1960 se atendieron en el Instituto Materno Infantil "Concepción Villaveces de Acosta" 20.338 partos, de los cuales correspondieron 234 a embarazos múltiples lo que da una incidencia de $1.13 \%$, siendo 4 de estos embarazos triples $(0,019 \%)$. No se presentó ningún embarazo de 4 o más fetos.

\section{$E D A D$}

La edad fluctúa entre los 15 y 41 años, encontrándose la mayoría de las pacientes entre 20 y 24 años, 71 casos (30,3\%) Fig. No 1.

\section{PARIDAD}

40 pacientes eran primigestantes $(17.2 \%), 49$ secundigestantes $(20 \%)$ y el resto multigestantes $(61.70 \%)$ Fig. No 2 . De éstas últimas, 3 tenían el antecedente de otro embarazo gemelar. $(1.3 \%)$. 


$$
\text { EDAD }
$$

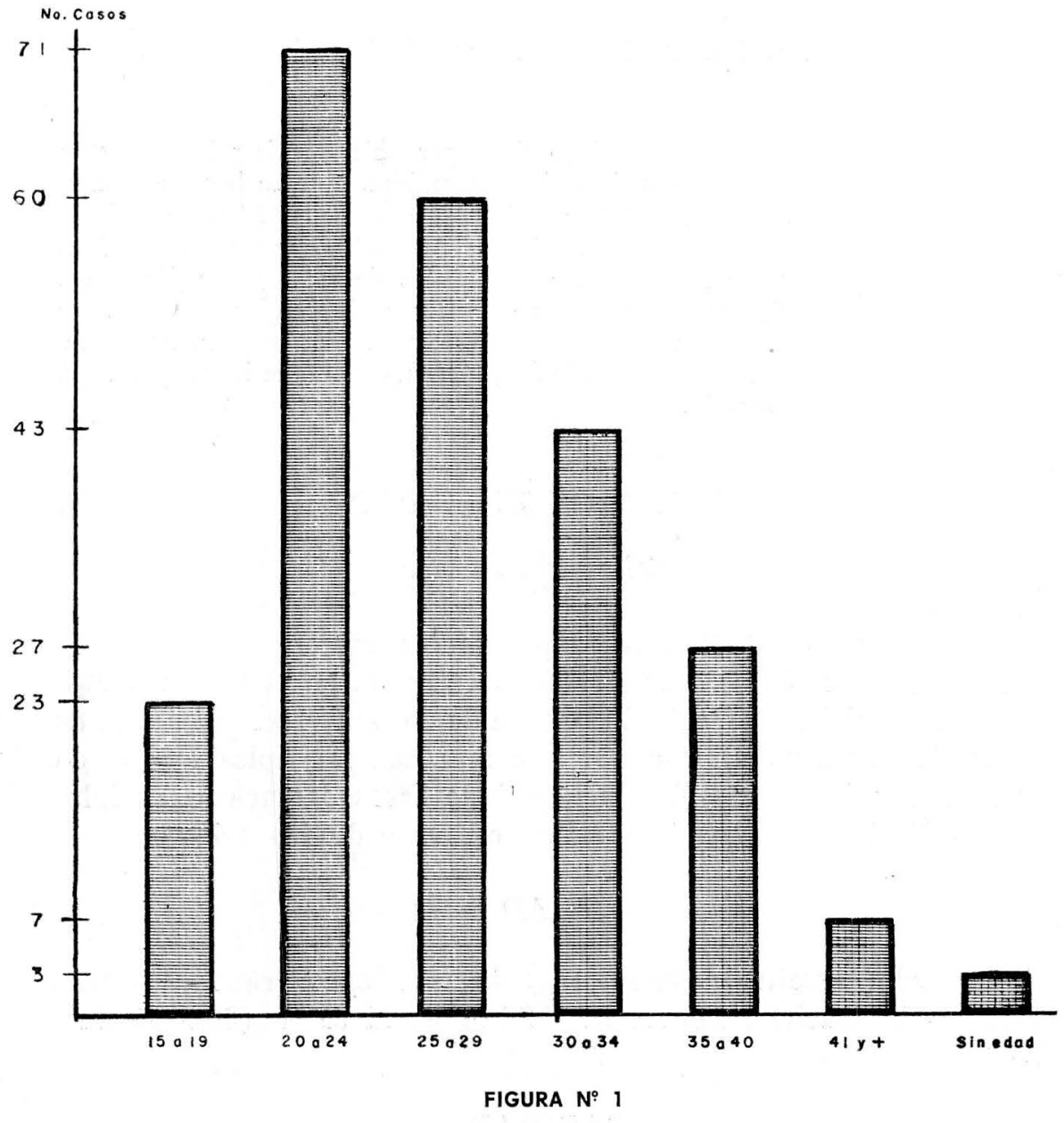


$P A R \mid D A D$

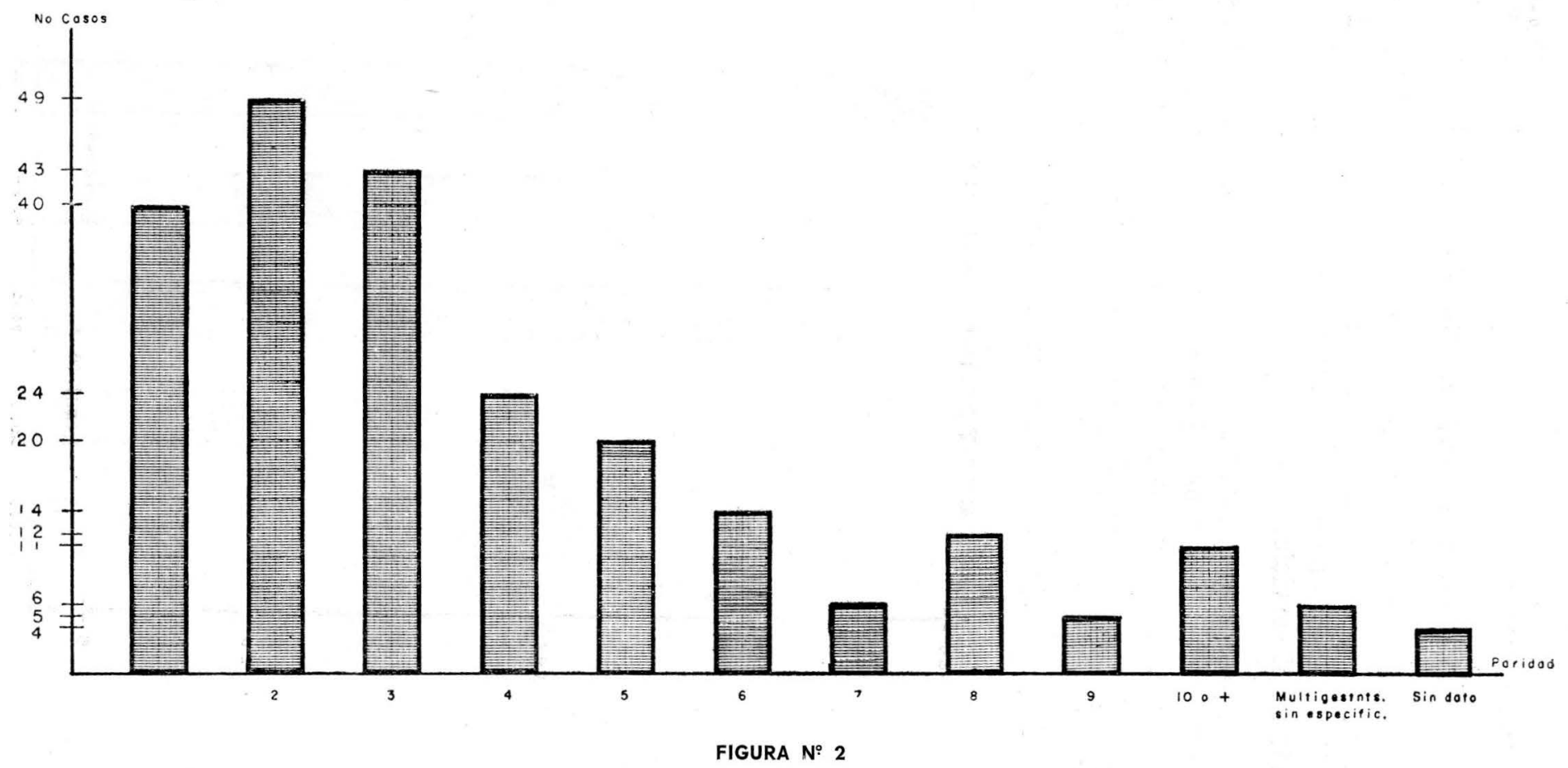




\section{DURACION DE LA GESTACION}

La duración de la gestación en semanas, en 139 pacientes, se encuentra especificada en la Fig. $\mathrm{N}^{\circ}$ 3. Aclaramos que de las 230, 86 figuran sin ningún dato al respecto y 5 casos no fueron atendidos en el servicio.

El promedio fue de 35 a 39 semanas, 74 casos $(32.2 \%)$, fluctuando entre 30 semanas el mínimo y 40 semanas el máximo. Autores americanos observan que la mayor frecuencia es de 33 a 36 semanas encontrando ellos dos casos de 41 o más semanas entre 203 embarazos múltiples. (9)

El promedio para los trillizos fué de 32 semanas.

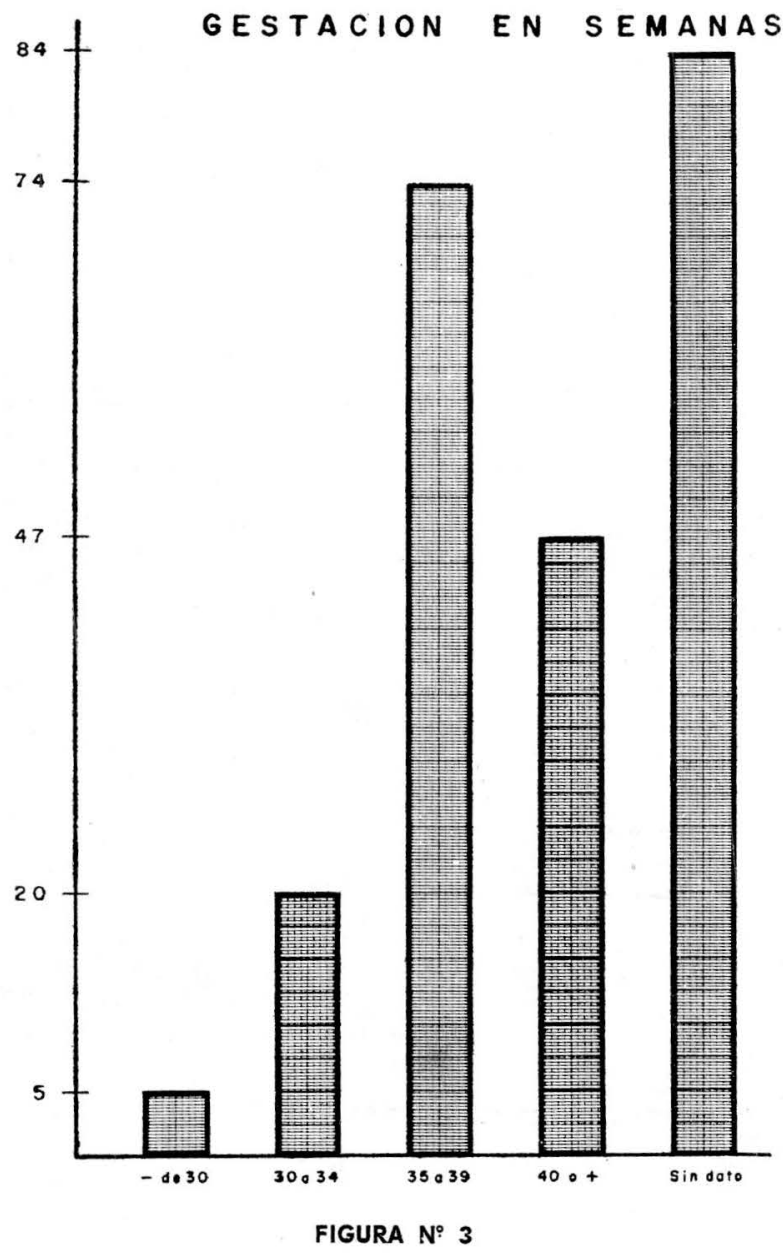




\section{DIAGNOSTICO}

Se hizo diagróstico positivo de embarazo gemelar en 51 casos, lo que equivale al $21.8 \%$ y sin diagnóstico 183 (78.2\%), contraste asombroso con las estadísticas americanas que tan solo anotan un 17 a $18 \%$. (8.9)

Esta anomalia se debe en gran parte a la falta de asistencia de las pacientes al servicio prenatal, pero más que todo a la poca observación del personal en los exámenes clínicos, pues de 24 pacientes que acudieron a prenatal tan solo se hizo el diagnóstico positivo en 14 de ellas.

De los 51 casos diagnosticados previamente, en 20 la confirmación se hizo mediante el empleo de Rayos X.

\section{ALTURA UTERINA}

Un $40.8 \%$ (94 casos) tenían una altura uterina de $35 \mathrm{cms}$. o más, siendo $25 \mathrm{cms}$. el mínimo y 45 el máximo.

\section{HIDRAMNIOS}

24 casos fueron diagnosticados como hidramnios, pero no consta en las Historias si se comprobó o no durante el parto. No podemos, pues, dar con precisión la incidencia de hidramnios y embarazo múltiple, que para algunos autores es de 8 a $9 \%$ (Waddell) (9).

\section{TOXEMIAS}

Entre los 234 casos de embarazo múltiple, 48 pacientes (20.7\%) fueron consideradas como preeclámpticas, aclarando que para catalogarlas como tal tuvimos en cuenta aquellas que presentaban a la vez presión arterial mayor de 140 x 90, edemas y proteinuria. La incidencia dada por autores americanos e ingleses fluctúa entre el 10.8 y el $37 \%$ (2-3-11). La cifra más baja, $10.8 \%$ consignada por Waddell y Hunter de la Clínica Mayo, se explica por el hecho de que casi todas sus pacientes asistieron a la consulta prenatal. De los 48 casos de toxemia tardía observados por nosotros, solamente 9 habían tenido control prenatal. 
Hubo un solo caso de eclampsia $(0.43 \%)$, manifestada durante el parto y el puerperio. Los autores atrás citados anotan dos casos de eclampsia del puerperio entre 203 embarazos múltiples $(1 \%)$. (9)

En 43 pacientes se observaron edemas marcados, sin que conste en las historias si había o no edema suprapúbico, signo casi constante en el embarazo múltiple. Con presión arterial alta, únicamente hubo 11 casos.

\section{PARTO}

Duración del trabajo: El promedio de horas de trabajo fué así: En primigestantes 13 horas para el primer gemelo y 14 horas para el segundo; en secundigestantes 8 horas 55 minutos para el primero y de 9 horas 40 minutos para el segundo; en multigestantes el promedio fue de 7 horas.

Como se ve, el intervalo del nacimiento del primero y el segundo fué por término medio en las primíparas, de una hora; en las secundigestantes de 45 minutos, fluctuando entre 5 el mínimo y 20 horas 30 minutos el máximo (tan solo un caso), no existiendo relación alguna entre el intervalo de nacimiento del primer feto $\mathrm{y}$ el segundo.

Ruptura precoz de membranas: Fué consignada en 5 casos $(2.2 \%)$, incidencia mucho más baja que la que dan ciertos autores $(13.3 \%)(9)$, lo cual se debe seguramente a la falta de observación y diagnóstico al ingreso de la paciente.

\section{PRESENTACIONES}

Hubo 233 casos de vértice $(54.1 \%)$ correspondiendo en su mayoría OIA (161 casos) y un solo caso de presentación de frente, cuyo parto se verificó con feto muerto después de haberse hecho mutación a cara.

En presentación de pelvis hubo 133 partos (31.2\%) siendo sensiblemente iguales la modalidad completa (68 casos) y la incompleta (65 casos). Hubo 23 casos de situación transversa del segundo gemelo $(5.1 \%)$ y 3 casos de ambos fetos en dicha situación. 
Tipo de parto: Sin contar los 22 casos atendidos fuera del servicio, 360 niños nacieron en parto espontáneo.

Por cesárea nacieron 24 fetos, 11 para ambos, habiéndose hecho el diagnóstico solo en 4 casos; y dos cesáreas para el segundo Las indicaciones fueron: eclampsia y pre-eclampsia, trabajo prolongado, placenta previa, situación transversa, sufrimiento fetal e iteractividad. Hubo necesidad de efectuar cesárea para el segundo feto en una situación transversa después de haber intentado la versión interna. En el otro caso se practicó la cesárea por falta de encajamiento.

Se hizo aplicación de fórceps en 13 casos.

Se practicó versión interna y extracción podálica en 22 casos.

De los 133 partos en preseritación de pelvis, 27 casos fueron auxiliados o corregidos con las maniobras de Moriceau o de Rojas.

En 13 casos hubo procidencia de cordón siendo la mayoría de ellas para el segundo gemelo (10 casos).

Se presentaron 38 casos de circulares del cordón no siendo posible verificar la presencia de entrecruzamientos o nudos verdaderos, que según casi todos los autores (6-7) se presenta en la mayoría de los gemelos monoamnióticos, y que son causa de muertes fetales dobles.

\section{SEXOS}

Hubo 70 casos en que ambos fetos fueron del sexo masculino y 75 en los que ambos fueron femeninos. En un caso de trillizos el sexo de todos fué femenino y en otro caso masculino. En el tercer caso, uno fué masculino y dos femeninos.

\section{PESOS}

El promedio de peso para el primer feto fué de 2.280 grs. y de 2.244 para el segundo, existiendo pesos extremos de 3.400 grs. 
el máximo y 800 el menor. El promedio de peso en los triliizos fue de 1.757 grs, fluctuando entre 2.200 y 1.345 grs.

\section{HEMORRAGIA POST-PARTO}

33 pacientes $(14 \%)$ presentaron hemorragia, siendo la causa más frecuente la atonía uterina. La pérdida sanguínea no fue posible de precisar pues ésta no se consigna en centímetros cúbicos sino en términos arbitrarios tales como: normal, escasa, abundante, notoria, intensa, etc. No hubo necesidad de practicar histerectomía por hemorragia ni parece haber existido ningún caso de coagulopatía, pues todos cedieron al tratamiento con ocitócicos. Dentro de este grupo de pacientes se presentaron 9 casos de hemorragia por retención de placenta y que cedió con la extracción manual respectiva.

\section{ANESTESIA}

Se suministró anestesia a 36 pacientes, siendo los agentes más empleados el éter ( 15 casos) para las versiones internas y la extracción manual de placenta y la anestesia raquídea (11 casos) para las cesáreas y fórceps. En los 10 casos restantes se utilizaron el ciclopropano, el trilene y el pentotal.

\section{CLASIFICACION}

Teniendo en cuenta su origen, el embarazo gemelar puede ser $\left.1^{9}\right)$ Univitelino (Uni-ovular Monozigota) cuando ambos huevos se desarrollan a expensas de un solo óvulo.

$2^{\circ}$ ) Bivitelinos (Biovular o Dizigota) cuando cada huevo proviene de un óvulo diferente. De acuerdo con los caracteres de la envoltura pueden ser: 1) Monocorial, cuando la envoltura corial es común a ambos huevos. 2) Bicorial, cuando cada huevo posee una envoltura completa (un amnios y un corion). (12)

$\mathrm{Al}$ revisar detenidamente las historias observamos que una de sus grandes fallas se encuentra en la clasificación seguida pues solamente en 13 casos, se encontraron datos suficientes para poderlos encajar en una nomenclatura internacional. Además se omitieron detalles importantes tales como el estado de la placen- 
ta, del amnios o del cordón. De estos 113 casos, 87 fueron bivitelinos biamnióticos $(76.9 \%), 23$ monovitelinos biamnióticos $(20.3 \%)$ y 3 casos de monoamnióticos univitelinos $(2.6 \%)$.

En los trillizos se presentaron dos casos de tricoriales triamnióticos. Un caso en que uno de los fetos venía con placenta y saco amniótico individuales y los otros dos fetos venían en un solo saco y con una sola placenta. El caso restante no está clasificado.

Es de anotar la alta incidencia de gemelos monoamnióticos, pues en 113 casos clasificados se presentaron 3. Revisando la literatura americana, encontramos una incidencia de un caso por cada 132 embarazos gemelares. Creemos, o nos asiste la duda, de que los 3 casos que figuran en nuestro estudio, no hayan sido bien clasificados, ya que es muy rara la doble supervivencia, tanto que hasta 1960 solo se han publicado 42 casos en la literatura mundial. (6-7) Si esos 3 casos fueran reales insistiríamos en una publicación especial, pues lo consideramos como un acontecimiento extraordinario.

\section{MORTALIDAD}

Materna. - En los 234 casos revisados no se presentó ningún caso de mortalidad materna.

Fetal. - Hubo 33 casos (66 fetos) en que murieron ambos post-parto, lo que equivale a un $14 \%$ de la mortalidad doble fetal. De éstos, 29 fueron del mismo sexo y 4 de sexo diferente. Hubo 32 fetos muertos, de los cuales 12 fueron para el primero y 26 para el segundo gemelo.

La mortalidad total Neonatal fué de 98 fetos, lo que da una incidencia de $22,6 \%$, cifra bastante alta si se le compara con la relatada en otros trabajos $(3,21 \%)(1-10)$.

Nacidos muertos hubo 8 casos para el primer feto y 18 para el segundo, dando un total de 26 casos, lo que equivale a $5.4 \%$.

Al relacionar la mortalidad con el peso de los fetos, notamos que la mayoría de las muertes se sucedieron por prematuridad $(71.4 \%$ ) como lo demuestra la Fig. No 4. En la mayoría de los 


\section{RELACION PESOS Y MORTALIDAD FETAL}

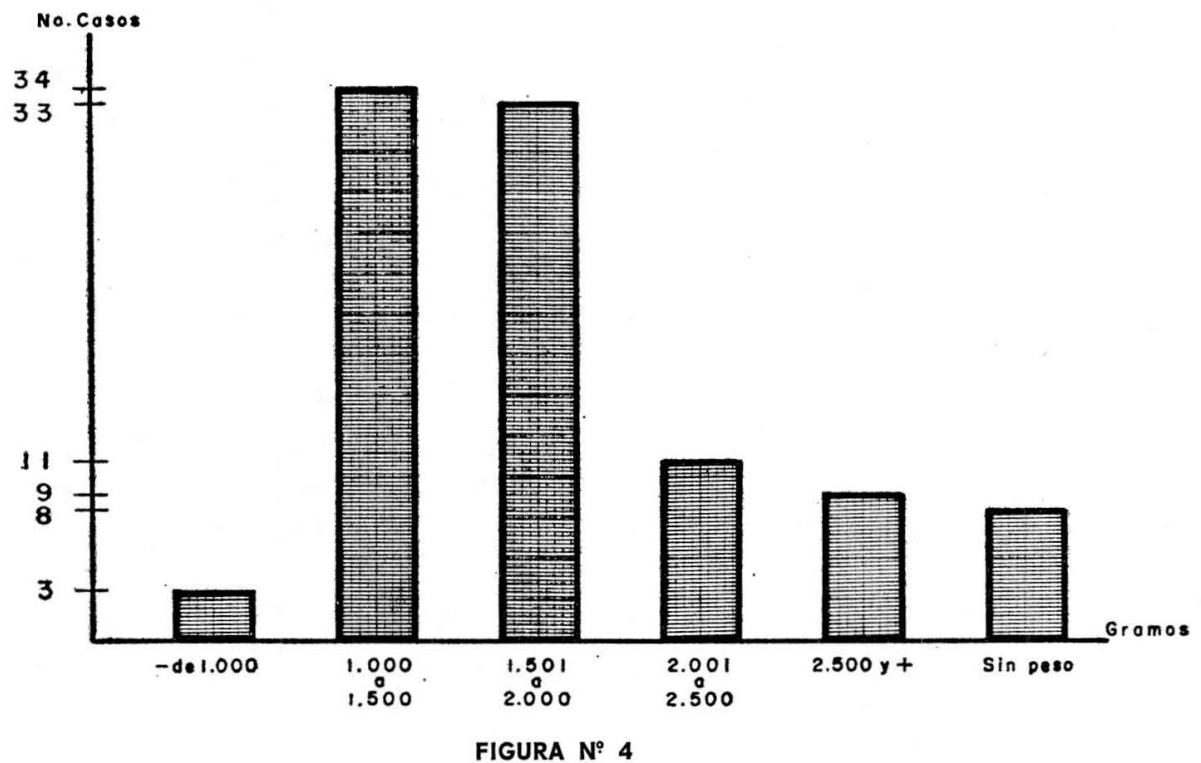

RELACION EDAD Y MORTALIDAD FETAL

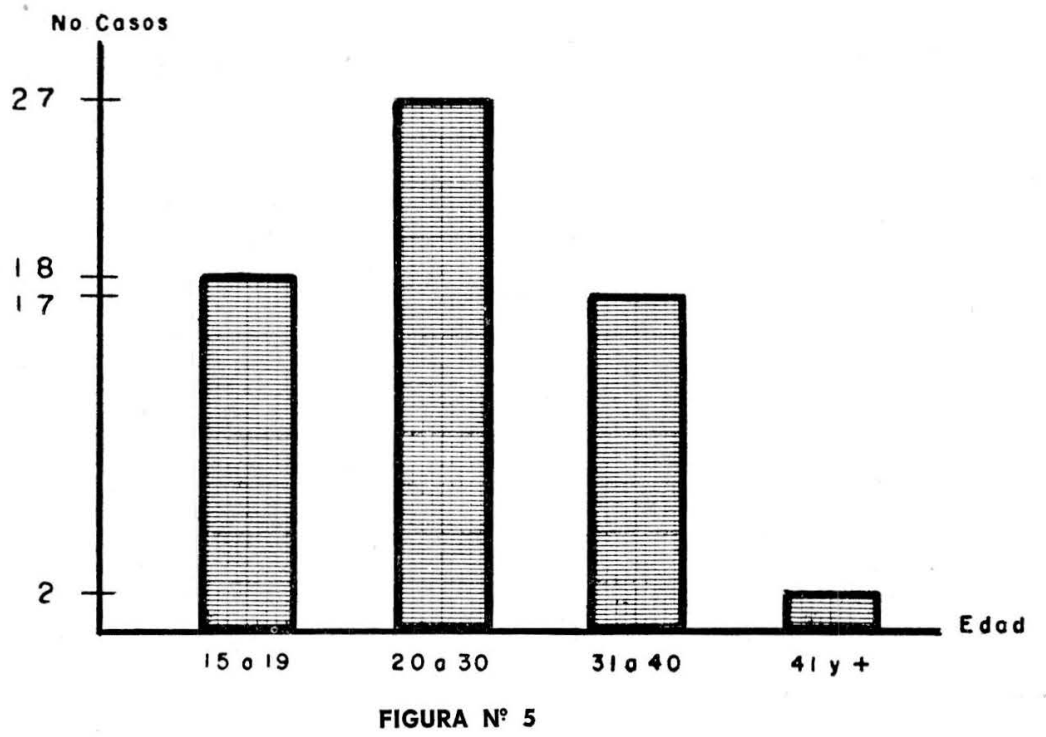


que pesaron más de 2.500 grms. su muerte se produjo por traumatismos obstétricos (versión externa, versión interna, maniobras de Rojas y Moriceau) o por presentaciones difíciles de conducir en el momento del parto. Nos llama la atención que entre más edad tenía la paciente, menos mortalidad fetal se produjo (Fig. No 5). Lo mismo sucedió con la paridad, siendo más frecuente la mortalidad entre los casos de primi y secundigestantes, que entre las multigestantes.

\section{RESUMEN}

Se hace la revisión de 234 casos de embarazo múltiple (incidencia de $1.13 \%$ ) atendidos en el I. M. I. en un lapso de 2 años. Cuatro de ellos fueron embarazos triples $(0.019 \%)$.

71 pacientes $(30.3 \%)$ tenían de 20 a 24 años. 40 eran primigestantes $(17.2 \%), 49$ secundigestantes $(20 \%)$ y el resto multigestantes $(61.7 \%)$.

La duración promedio de la gestación fué de 35 a 39 semanas.

El diagnóstico previo tan solo se hizo en 51 casos $(21.8 \%)$ 20 con la ayuda de Rayos X.

La incidencia de pre-eclampsia fué de $20.7 \%$ (48 casos). Hubo un solo caso de eclampsia $(0.43 \%)$.

El promedio de trabajo de parto fué de 13 horas para las primigestantes; de 8 horas 55 minutos, para las secundigestantes; y de 7 horas para las multigestantes.

233 partos se verificaron en presentación de vértice $(54.1 \%)$ y uno en presentación de cara. 133 casos en presentación podálica $(31.2 \%)$. Hubo 23 casos de situación transversa del segundo gemelo y 3 de ambos fetos.

Por cesárea nacieron 24 fetos, de los cuales 2 eran segundos gemelos. Se hizo aplicación de fórceps en 13 casos. Se hizo versión interna seguida de extracción podálica en 22 casos. 
De 13 casos de procidencia del cordón, 10 se presentaron en el segundo gemelo.

En 70 casos ambos fetos eran del sexo masculino y en 75 eran del sexo femenino.

El promedio de peso para el primer feto fue de $2.280 \mathrm{gr}$. y de 2.244 para el segundo. El promedio de peso para los triliizos fue de 1.757 grs.

El $14 \%$ de las pacientes (33 casos) presentaron hemorragia de post-parto, casi todas por atonía uterina.

A 36 pacientes se les suministró anestesia, siendo el éter el agente más empleado (15 casos) y luego la anestesia raquídea (11 casos).

Solamente en 113 casos se encontraron datos suficientes en las historias para poder clasificar los embarazos múitiples según su origen. 87 se consideraron bivitelinos-biamnióticos, 23 monovitelinos-biamnióticos y 3 monoamnióticos-univitelinos.

No hubo mortalidad materna.

La mortalidad total neonatal fue de 98 fetos $(22.6 \%)$ de los cuales 26 nacieron muertos. En 33 casos murieron ambos fetos (14\% de doble mortalidad). En 32 casos murió uno de los fetos, correspondiendo 12 al primero y 20 al segundo.

Solo un niño sobrevivió, de 3 de los 4 casos de embarazo triple. En el otro se desconoce su estado.

El $71.4 \%$ de los fetos murieron por prematuridad. En los de término, la mayoría murió por traumatismo obstétrico.

\section{CONCLUSIONES}

$1^{\text {a }}$ - Con miras a facilitar y hacer más exactos los estudios estadísticos que en el futuro vayan a elaborarse, es imperativo que las historias clínicas se elaboren en forma adecuada. 
$2^{\mathrm{a}}$ - Es necesario que se agudice el criterio clínico-obstétrico en la consulta prenatal y al ingresar las pacientes al Instituto, para evitar que las sorprenda el parto sin haberse hecho el diagnóstico de embarazo múltiple, descuido éste que influye en la mortalidad fetal. Creemos que el empleo de los Rayos X debe conservar su vigencia cuando se trate de confirmar un diagnóstico de gestación múltiple.

$3^{a}$ - Deberá insistirse en la educación prenatal de las pacientes hospitalarias para reducir con ella la elevada incidencia de toxemia.

$4^{\mathrm{a}}$ - Confirmado, y aún sospechado el embarazo múltiple, deberán las pacientes vigilarse atentamente durante todo el trabajo de parto pensando siempre que no se trata de un caso obstétrico común y corriente.

$5^{\mathrm{a}}$ - La atención del parto múltiple tiene que encomendarse al personal asistencial más experimentado ya que en un alto porcentaje habrá de requerirse el auxilio, la corrección o la intervención quirúrgica.

$6^{\text {a }}$ - Siendo el promedio del peso fetal en los casos de embarazo múltiple inferior a 2.300 grms., en la conducción y atención del parto se tendrá en cuenta todos los cuidados que se deben prodigar a los niños prematuros.

\section{BIBLIOGRAFIA}

1 MADELENE M. DONNELLY (Des Moines Iowa.) The Influence of Multiple Butte on perinatal Loss. Am. J. Obst. \& Gynec. 72998 1.003 - 1956.

2 POTTER E., LAND CRUDEN. 'Twin pregnancies' in the service of the Chicago Lying in Hospital. Am. J. Obst. \& Gynec. 428701941.

3 POTTER and FULLER. Am. J. Obst. \& Gynec. 581391949. 581391949.

4 HIRST J. C. Maternal and fetal expectations with multiple pregnancy. Am. J. Obst. \& Gynec. 376341939. 
5 GUTTMACHER A. F. An annalysis of 521 cases of twin pregnancies. Am. J, Obst. \& Gynec. 341937.

6 TAFEEN CURL H. FREEDMAN HEURY L. and KABANE ALBERT J. Monoamniotic Twins. Am. J. Obst. \& Gynec. 791.0781 .0811960$.

7 GREM OVENTIN L. JACKSON JAMES and MILLER MILLER ALEXANDER. Monoamniotic Twins. Am. J. Obst. \& Gynec. 771.082 1.0841960 .

8 LIENT CHARLES A. NOVOTY (M. C.) U. S. Navy Lieut, WILLIAM K. HASS (M. C.) Naval Reserve U. S. Navy Capt. DWIGHT S. CALLAGAU (M. C.) U. S. Navy Early diagnosis of multiple pregnancy. Jama. 171880417 October 1959.

9 KENNETH E. WADDELL, M. D. JAMES S. HUNTER, Jr. M. D. Twin pregnancies Review of 203 cases. Am. J. Obst. \& Gynec. 80: 756-760 1960.

10 AARON J. B. and HALPERIN, JACOBS. Fetal survival in 376 twin deliveries. Am. J. Obst. \& Gynec. 697941955.

11 BULFIN M. J. and LAROLER P. E. Problems associated with toxemia in twin pregnancies. Am. J. Obst. \& Gynec. 73371957.

12 LEON JUAN. Tratado de Obstetricia. 22603101957.

13 ZERDA JIMENEZ LEOPOLDO Tesis de Grado U. N. 1948. "Anotaciones Clínicas y Estadísticas sobre Embarazo y Parto Múltiple”. 